\title{
Implication of Porphyromonas gingivalis in colitis and homeostasis of intestinal epithelium
}

\author{
Yoojin Seo ${ }^{1,2}$, Su-Jeong Oh ${ }^{1,2}$, Ji-Su Ahn ${ }^{1,2}$, Ye Young Shin ${ }^{1,2}$, Ji Won Yang ${ }^{1,2}$ and Hyung-Sik Kim ${ }^{1,2^{*}}$
}

\begin{abstract}
Emerging evidences have reported that periodontitis can be a risk factor for the pathogenesis of various systemic diseases. Porphyromonas gingivalis $(\mathrm{Pg})$, one of the crucial pathogens in chronic periodontitis, has been spotlighted as a potential cause for the promotion and acceleration of periodontitis-associated systemic disorders. To investigate the association between $\mathrm{Pg}$ and intestinal disease or homeostasis, we treated Pg-derived lipopolysaccharide (LPS) in murine colitis model or intestinal organoid, respectively. Pg-derived LPS (Pg LPS) was administrated into chemically induced murine colitis model and disease symptoms were monitored compared with the infusion of LPS derived from E. coli (EC LPS). Organoids isolated and cultured from mouse small intestine were treated with Pg or EC LPS and further analyzed for the generation and composition of organoids. In vivo observations demonstrated that both $P g$ and EC LPS exerted slight protective effects against murine colitis. Pg LPS did not affect the generation and growth of intestinal epithelial organoids. Among subtypes of epithelial cells, markers for stem cells, goblet cells or Paneth cells were changed in response to Pg LPS. Taken together, these results indicate that $\mathrm{Pg}$ LPS leads to partial improvement in colitis and that its treatment does not significantly affect the self-organization of intestinal organoids but may regulate the epithelial composition.
\end{abstract}

Keywords: Periodontitis, Porphyromonas gingivalis, Colitis, Intestinal epithelium, Organoid

\section{Introduction}

Periodontitis is a chronic oral inflammation which destroys the tooth-supporting periodontium including gingiva, alveolar bone and periodontal ligament [1]. This disease is one of the most prevalent chronic diseases. Over the decades, several bacteria have been reported to be associated with periodontitis [2]. In particular, Porphyromonas gingivalis $(P g)$ is considered as one of the crucial pathogenic bacteria for periodontitis [3-10]. A number of studies have proposed that $P g$ is involved in the onset and progression of extra-oral inflammatory disorders, such as diabetes, cardiovascular diseases, rheumatoid arthritis, adverse pregnancy outcomes and pulmonary diseases [11-15]. More recently, it has been demonstrated that periodontitis might be associated with

\footnotetext{
* Correspondence: hskimcell@pusan.ac.kr

${ }^{1}$ Department of Life Science in Dentistry, School of Dentistry, Pusan National University, Busandaehak-ro 49, Yangsan 50612, Republic of Korea

${ }^{2}$ Dental and Life Science Institute, Pusan National University, Yangsan 50612, Republic of Korea
}

inflammatory bowel diseases (IBD) $[16,17]$. IBD describes a group of inflammatory conditions of the gastrointestinal tract and can be categorized into two major forms, Crohn's disease and ulcerative colitis [18]. Although periodontitis and IBD might share similar etiologic pathways and large quantities of oral bacteria can reach the intestine through saliva flow [19], only few studies have experimentally reported the association between periodontitis-exacerbating pathogens and IBD [20, 21]. Therefore, in the present study, we sought to investigate the impact of Pg LPS in mouse colitis model induced by dextran sulfate sodium (DSS).

The epithelium of the digestive tract provides a large surface area for interaction of host and microbiota and serves as a primary balance system for tolerance and pathogen clearance [22]. The intestinal epithelium exhibits rapid turnover in $4-5$ days and this robust regenerative capacity depends on stem cells residing in the bottom of crypts between villi [23]. Intestinal stem cells (ISCs) critically contribute to the homeostasis of gut 
epithelium by generating five epithelial cells, including enterocytes, goblet cells, Paneth cells, tuft cells and enteroendocrine cells. A number of studies have uncovered that stem cells from various tissues can directly recognize pathogen-associated molecular patterns (PAMPs) and their function including self-renewal and differentiation can be altered in response to PAMPs [24-27]. Given that oral cavity and gastrointestinal tract are directly linked and oral bacteria or their components can be frequently exposed to intestinal epithelium via saliva flow, we investigated the influence of $P g$ LPS on functions of ISCs. We utilized three-dimensional organoid culture models to assess the direct impact of $P g$ LPS on epithelial homeostasis or regeneration with the exception of other cell types in the intestine including immune cells or stromal cells which also respond to microbial components and subsequently regulate epithelial maintenance [28].

\section{Results}

Pg LPS exerts modest beneficial effects against DSSinduced colitis in mice

We first investigated whether the administration of $P g$ LPS can affect the severity of DSS-induced colitis. 3\% DSS was added in drinking for 7 days to induce acute colitis and $P g$ LPS was orally infused at day 1, 3, 5 and 7. Interestingly, oral administration of Pg LPS or Ec LPS slightly ameliorated the loss of body weight and rescued mice from lethality compared to PBS-treated group (Fig. 1a). On day 12, the disease activity index (DAI) was significantly decreased in $P g$ or Ec LPS-treated group (Fig. 1b). After the assessment of DAI, the mice were sacrificed for gross and histological evaluation of colonic inflammation and damage. Colon length was reduced in PBS-treated group, however, it was moderately restored by $P g$ or Ec LPS treatment (Fig. 2a). H\&E staining and histopathological analysis revealed that DSS treatment led to the destruction of epithelium and infiltration of inflammatory cells, and the administration of both $P g$ and Ec LPS significantly attenuated theses epithelial damages observed in colons of colitic mice (Fig. 2b). As expected from gross observations, the administration of both $P g$ and Ec LPS significantly attenuated the destruction of the epithelial structure, as well as the infiltration of lymphocytes (Fig. 2b). Taken together, our results suggest that $P g$ LPS exhibits moderate protective effects in chemically induced colitis of mice and its effect and potency are similar to Ec LPS.

\section{Pg LPS does not affect the morphology or growth of} mouse intestinal organoids but regulates the expression of genes for epithelial cells

In order to specifically analyze the impact of $P g$ LPS on intestinal epithelial cells, we generated 3D culture organoids from mouse small intestine and LPS was treated. Mouse small intestinal organoid (SIO) showed a spherical structure with appearance of surface protrusions as it grows (Fig. 3a). LPS from $P g$ or $E c$ did not alter the morphology of SIO nor the formation of budding structure (Fig. 3a and b). We then explored whether the differentiation of epithelial cells is regulated by Pg LPS by quantitative PCR of selected representative genes for each epithelial cell subtype. The expression of Bmi1, a marker for quiescent ISCs (Q-ISC), was not affected by $P g$ or $E c$ LPS treatment. Among markers of active stem cells, $L g r 5$ expression was significantly down-regulated after $P g$ LPS treatment whereas EphB2 expression was up-regulated (Fig. 4a). The expression of markers for the proliferation was not affected (Fig. 4a). $P g$ and Ec LPS treatment significantly elevated the expression of

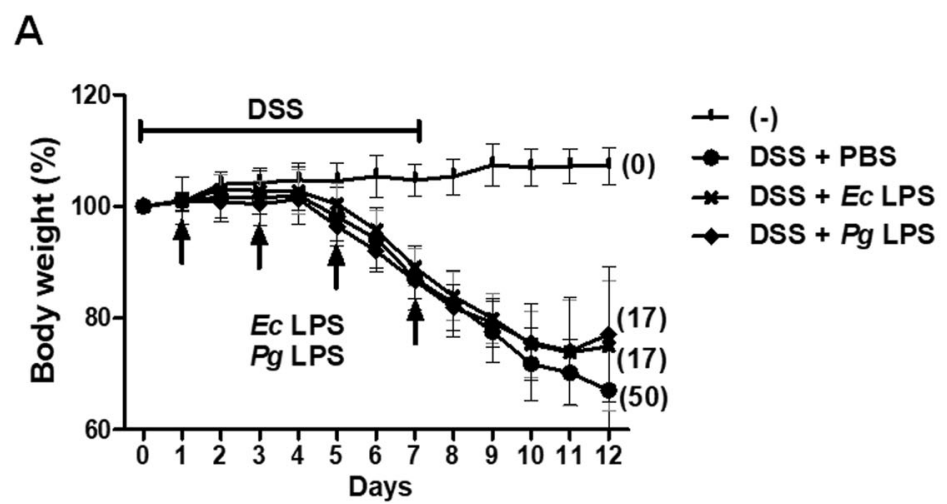

B

Fig. 1 Monitoring of colitic mice. (a) Body weight loss and lethality of mice were monitored for 12 days. The body weight of each mouse at day 0 was calculated as 100\%. Numbers in parentheses mean the percentage of dead mice. In 'DSS + PBS' group, 2 mice at day 11 and 1 mouse at day 12 died. In 'DSS + ECLPS' and 'DSS + EcLPS' group, 1 mouse at day12 died, respectively. (b) Disease activity index indicating colitis severity was scored at day 12.6 mice per group were used. In (b), $p$-value significance was calculated by comparing other groups against $(+)$ group (marked as + ). ${ }^{* *} P<0.01$ 


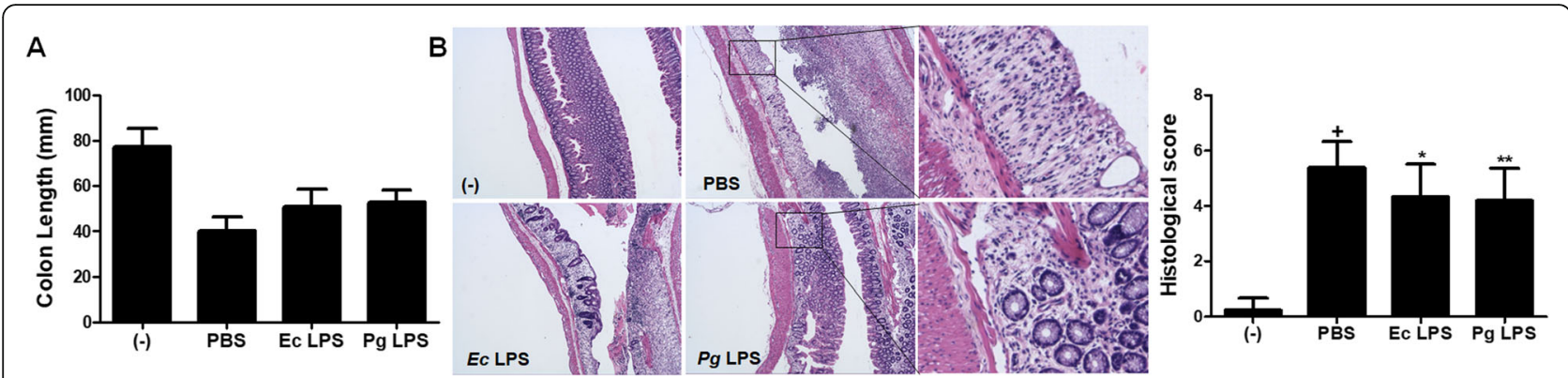

Fig. 2 Gross and histological evaluation of colon. (a) Colon length was measured from proximal colon to rectum. (b) Destruction of epithelial structure and scattered infiltration of inflammatory cells were assessed as histopathological score in H\&E stained section of colon tissues. Results are shown as mean \pm SD. Representative areas showing the damaged area with typical structure destruction and leukocyte infiltration (black box) were magnified. In (b), p-value significance was calculated by comparing other groups against (+) group (marked as + ). ${ }^{*} P<0.05$, ${ }^{* *} P<0.01$

Defensin, a marker for Paneth cells, however, only Ec LPS significantly increased the expression of another Paneth cell marker, Lysozyme (Fig. 4b). One of markers for enterocytes, Alpi was reduced to a significant extent in response to $P g$ LPS (Fig. 4b). In addition, the expression of TTF, a goblet cell marker, was significantly upregulated by LPS from both bacteria (Fig. 4b). Lastly, the differentiation of enteroendocrine cells (EECs) was not affected when determined by Neurogenin 3 expression (Fig. 4b). These results indicate that Pg LPS does not critically affect the morphology or growth of mouse SIO, but possibly regulates the differentiation of subtype cells which constitute intestinal epithelium based on the determination of changes in marker expression for stem cells, Paneth cells, enterocytes and goblet cells.

\section{Discussion}

In the present study, we investigated the influence of LPS from $P g$, a keystone bacterium in periodontitis, in intestinal inflammation and homeostasis. The inflammation initiated by periodontitis can lead to systemic inflammation, therefore, it is plausible to presume that periodontal diseases might exacerbate IBD symptoms. A study by Arimatsu et al. has demonstrated that oral administration of $P g$ changed the composition of gut microbiota, as well as the function of gut epithelial barrier [20]. Moreover, in the study, insulin resistance was elevated in $P g$-fed mice. In another study, $P g$ administration damaged tight junction in small intestine and bacterial influx was followed. In addition, IL-6 level in small intestine and TNF- $\alpha$ level in large intestine were significantly elevated [21]. Recently, Blasco-Baque et al. established a murine model of periodontitis by infecting periodontium with Pg, Fusobacterium nucleatum and Prevotella intermedia. They observed modest or subtle changes of gut microbiota [29]. However, none of these studies investigated simple direct impact of $P g$ LPS in DSS-induced murine colitis model. In the present study, although we expected that the oral administration of $P g$ LPS might accelerate or aggravate colitis symptoms, however, on the contrary, Pg LPS treatment exerted modest protective effects against colitic mice. Ec LPS treatment showed similar effects. Saito et al. reported that the administration of Ec LPS significantly suppressed colonic inflammation induced by DSS. They also suggested that repeated stimulation with LPS reduced

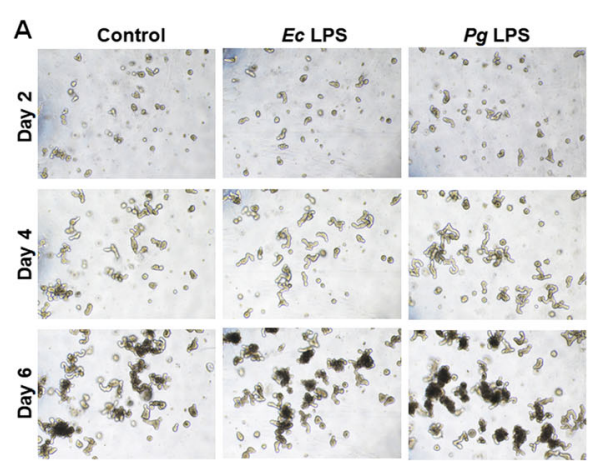

B

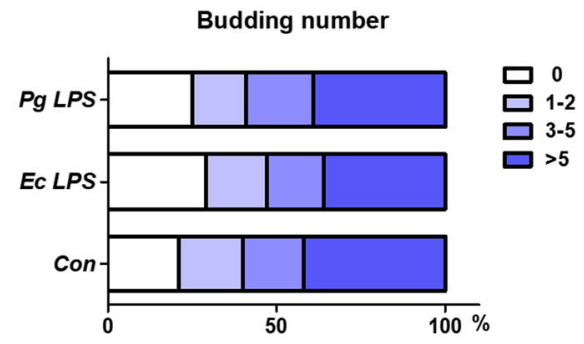

Fig. 3 Observation of the organoid from mouse small intestine. The organoid was generated from crypt cells of moue small intestine and maintained using conditioned media (a) Photographs of organoids were taken at day 2, 4 and 6 in the presence or absence of LPS. (b) The number of budding was counted on day 5 


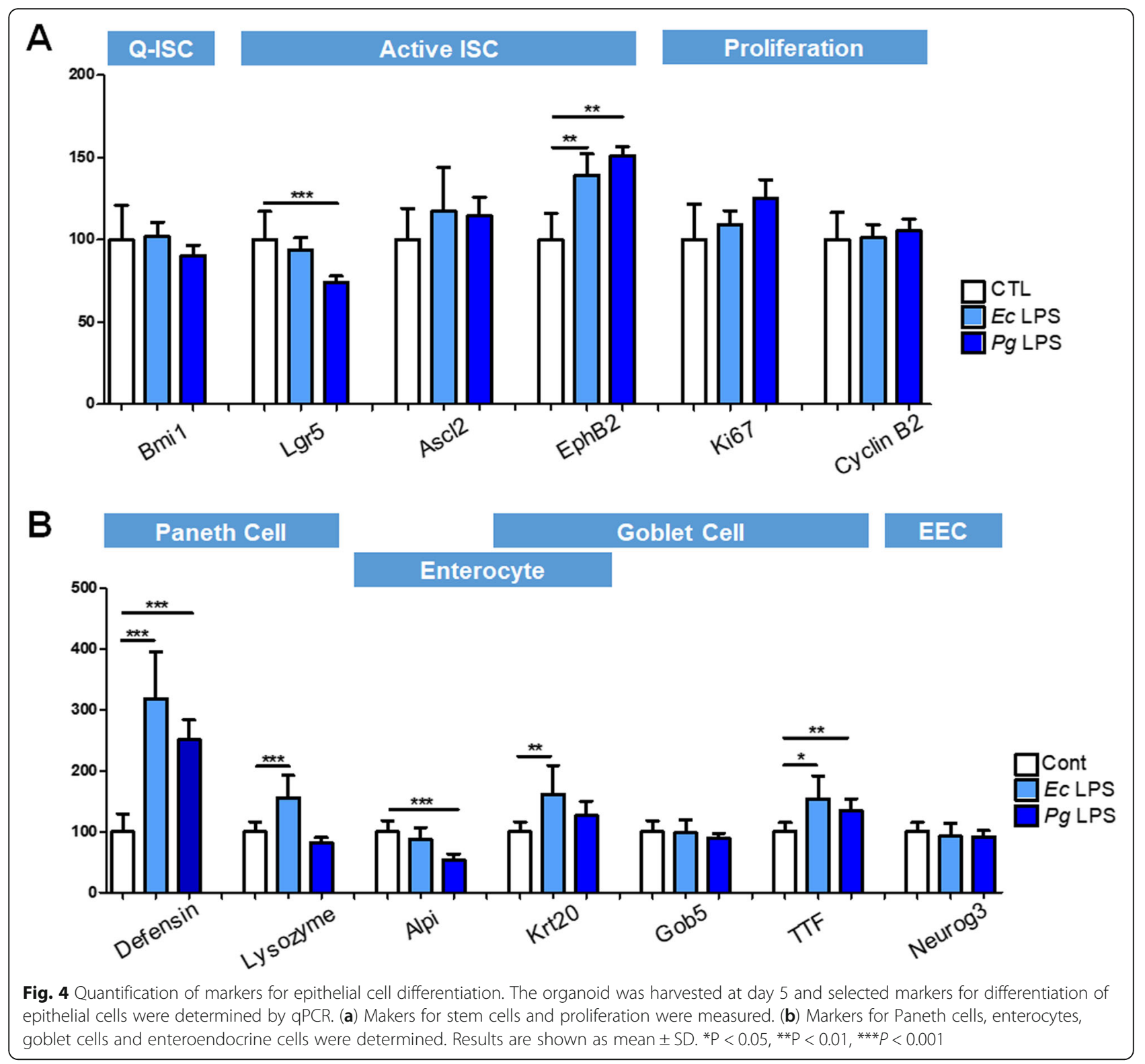

TNF- $\alpha$ production and up-regulated a negative regulator of Toll-like receptor (TLR) 4 signaling [30]. Along with this study, our present study proposes that $P g$ LPS might act through similar mechanisms or signaling as Ec LPS, at least in DSS-induced colitis. This observation supports the currently accepted theory that intestinal homeostasis is mainly maintained by TLR signaling, in spite of the continuous exposure to microbiota or their derivatives [31-33].

Among TLRs, the receptor for $P g$ LPS has been reported as TLR2, which is still controversial. Several studies have suggested that $P g$ LPS exhibits its activity via TLR2 [34, 35]. However, Ogawa et al. revealed that $P g$ LPS activates cells through TLR4 and not TLR2 by investigating the structure and function of lipid $\mathrm{A}$ of $\mathrm{Pg}$
LPS [36]. In addition, Hirschfeld et al. proposed that TLR2 activation of Pg LPS might be mediated by contaminated lipoprotein [37]. Therefore, it is still controversial through which $P g$ LPS exerts its activities. A recent study by Nativel et al. demonstrated that $P g$ LPS initiates pro-inflammatory signaling exclusively via TLR4 [38]. On the contrary, Andrukhov etl al. showed that $P g$ LPS and Ec LPS differently regulated the pattern of cytokine production from human gingival fibroblasts [39]. In the present study, we tried to investigate whether LPS from $P g$ or $E c$ differently regulates the function of intestinal stem cells using organoid culture system. Although the morphology, as well as the growth of the organoids were not affected by both LPS, Pg LPS exhibited different activities on the differentiation of epithelial cells 
compared with Ec LPS. Particularly, only Pg LPS downregulated the expression of $\operatorname{Lgr} 5$, a representative marker for intestinal stem cells, and Alpi, a marker for absorptive enterocytes. Our finding is consistent with the results from Nalto et al. suggesting that LPS from different microbiota (Acinetobater, Delftia and Stenotrophonas) differently regulate the differentiation of epithelial cells by acting on $\operatorname{Lgr} 5^{+}$stem cells [40].

Our study has its limitation in that $P g$ LPS action in colitis was determined using only one chemically induced model. To provide convincing evidences for the association between periodontitis and IBD, future studies using a number of models including recently established CRISPR/Cas9-mediated knockout mice with intestinal phenotypes [41-43], might be required. In addition, single cell RNA sequencing for organoid analysis can provide more visualized trajectory exploration for epithelial differentiation.

\section{Conclusions}

In conclusion, the present study indicates that LPS from periodontal pathogen, Porphyromonas gingivalis, exerts disease-ameliorating activities in chemically induced murine colitis model. We also found that Porphyromonas gingivalis-derived LPS differently regulates the lineage differentiation of intestinal epithelial cells compared to E. coli-derived LPS, whereas both LPS does not affect general growth and maturation of organoids mediated by intestinal stem cells.

\section{Materials and methods}

\section{Colitis induction and evaluation}

Animal experiments were conducted according to the approval and regulations of the Institute of Laboratory Animals Resources (PNU-2018-2034, Pusan National University). Experimental colitis was induced in mice by the addition of 3\% (weight/volume) DSS (MP Biochemicals, Solon, $\mathrm{OH}$ ) was added in drinking water for 7 days to induce the experimental colitis in $\mathrm{C} 57 \mathrm{BL} / 6$ mice (male, 8-10 wks) [29]. Animals were divided into 4 groups as negative (normal drinking water, $n=6$ ) control and vehicle (DSS + PBS, $\mathrm{n}=6)$ controls and Ec LPS $(10 \mu \mathrm{g} /$ head, $\mathrm{n}=6)$ and $P g$ LPS $(10 \mu \mathrm{g} / \mathrm{head}, \mathrm{n}=6)$ treated group. LPS was administrated at day 1, 3, 5 and 7 per oral administration. Mice were monitored for 12 days for body weight and survival rate. On day 12, colitis severity of mice was measured by scoring disease activity index. All animals were sacrificed followed by the gross examination of colons and further histopathological evaluation using $H \& E$ staining.

\section{Colitis severity}

Colitis severity was determined by scoring the disease activity index on stool consistency (graded from $0 \sim 4$ ), rectal bleeding $(0 \sim 4)$, coat roughness $(0 \sim 4)$, and general activity $(0 \sim 2)$.

\section{Histopathological examination}

Colon samples were prepared, fixed in formalin, processed in cycles of alcohol-xylene changes, and embedded in paraffin. $5 \mu \mathrm{m}$ sections of colon samples were prepared and stained with H\&E. The infiltration of lymphocytes (graded from $0 \sim 4$ ) and damages of intestinal epithelium (graded from $0 \sim 4$ based on the measurement of damaged area) were graded blindly.

\section{Small intestinal organoid (SIO) culture}

Murine small intestinal organoids were established with Intesticult organoid system (Stemcell Technologies, Vancouver, Canada) as manufacturer's instruction with minor modification. Briefly, the proximal part $(\sim 10 \mathrm{~cm})$ of duodenum was isolated and flushed with cold PBS 3 times. SI was then incised longitudinally and the intestinal villi and mucus were scraped off using a coverslip. Tissue was cut into $2-3 \mathrm{~mm}$ segments and collected into $50 \mathrm{ml}$ tube containing cold PBS. The fragments were washed with vigorous shaking and settled down, followed by supernatant removal. This washing step was repeated until the supernatant was clear (usually 3-5 times). Crypts were detached using Gentle Cell Dissociation Reagent (Stemcell Technologies) by 20-min-long incubation at room temperature with gentle rocking ( $20 \mathrm{rpm}$ ). After filtration with $70 \mu \mathrm{m}$ cell strainer (BD Falcon, Franklin Lakes, NJ). Flow-through crypts were centrifuged at $290 \mathrm{~g}$ for $5 \mathrm{~min}$. Pellets were resuspended in DMEM/F12 (Gibco, Grand island, NY) then centrifuged at $200 \mathrm{~g}$ for $5 \mathrm{~min}$. Approximately 250 crypts were resuspended in $20 \mu \mathrm{l}$ of DMEM/F12 and $20 \mu \mathrm{l}$ of Matrigel (Corning Life Sciences, Corning, NY) and total $40 \mu \mathrm{L}$ of crypt-Matrigel suspension was seeded into each well of 24 well plate followed by incubation at $37^{\circ} \mathrm{C}$ for $30 \mathrm{~min}$. After polymerization of the Matrigel, $750 \mu \mathrm{l}$ of IntestiCult $^{\mathrm{TM}}$ Organoid Growth Medium (Stemcell Technologies) was added for culture maintenance with or without $P g$ or Ec LPS $(10 \mu \mathrm{g} / \mathrm{mL})$. At day 5, the budding number of organoids were calculated, followed by the harvesting and further lysis for RNA isolation.

\section{RNA isolation and quantitative real-time PCR (qPCR)}

Total RNA of SIOs were extracted with RNeasy Mini Kit (Qiagen, Hilden, Germany) and cDNA was synthesized using ReverTra Ace ${ }^{\oplus}$ qPCR RT Master Mix (Toyobo, Osaka, Japan) as manufacturer's instruction. The quantitative PCR (qPCR) was performed using SYBR Green reagents (Thermo Scientific) on a ABI 7500 real-time PCR instrument (Applied Biosystems, Carlsbad, CA). Primer sequences are summarized in Table 1 . Relative expression 
Table 1 Primer sequences for qPCR

\begin{tabular}{lll}
\hline Gene & Forward primer & Reverse primer \\
\hline Lgr5 & GGGAGCGTCACGGGCCTTC & GGTTGGCATCTAGGCGCAGGG \\
Bmi1 & CCGGGATCTITATCAAGCA & TACCCTCCACACAGGACACA \\
Ascl2 & CATGGAAGCACACCTTGACTG & CATCAAGCTTGCATTCAGCC \\
Ephb2 & CAACGGTGTGATCCTGGACTAC & CACCTGGAAGACATAGATGGCG \\
Lysozyme & TGAACGTTGTAGTTGCCA & TGAGCTAAACACACCCAGTCG \\
TFF3 & TAATGCTGTTGGTGGTCCTG & CAGCCACGGTTGTACACTG \\
Alpi & GTGCCAAGAAGCTGCAGCCCA & GGCTAGGTGTCTCCGGTCC \\
Neurog3 & TITGTAAGTTTGGCGTCATC \\
Ki67 & GCATGCACAACCTCAACTC & GGCTTCTCATCTGTTGCTTCCT \\
CyclinB2 & CCTTGCTGTCCCCGAAGA & CAGAGCTGGTACTTGGTGTC \\
a-defensin-5 & GCCAAGAGCCATGTGACTATC & ACGCGTCTCTTCTITGCAGCC \\
Krt20 & ACTGAGGAGCAGCCAGGGGA & TCTAGGTTGCGCTCCAGAGACT \\
Gob5 & GGATTCGAGGTTCAAGTCACGG & AAGAGACTAAAACTGAGGAGCAGC
\end{tabular}

levels of each marker were normalized to Gapdh expression.

\section{Statistical analysis}

The mean value of the different groups was demonstrated as the mean $\pm \mathrm{SD}$. All statistical comparisons were performed using one or two-way ANOVA followed by the Bonferroni post-hoc test for multi-group comparisons using the GraphPad Prism version 5.01 (GraphPad Software, San Diego, CA). Statistical significance designated as asterisks is indicated in the figure legends.

\section{Abbreviations}

DAl: disease activity index; DSS: dextran sulfate sodium; EEC: enteroendocrine cell; IBD: inflammatory bowel diseases; ISCs: intestinal stem cells; LPS: lipopolysaccharide; PAMPs: pathogen-associated molecular patterns; Pg: Porphyromonas gingivalis; Q-ISC: quiescent intestinal stem cell; SIO: small intestinal organoid

\section{Acknowledgements}

Not applicable.

\section{Authors' contributions}

Y.S. designed the study, collected and analyzed the data, and wrote the manuscript; S-J.O., J-S.A., YY.S. and JW.Y collected and analyzed the data; HS.K. designed and supervised the study, analyzed the data and wrote the manuscript.

\section{Funding}

This work was supported by a 2-Year Research Grant of Pusan National University.

\section{Availability of data and materials}

All data produced or analyzed in the present study are available upon reasonable request.

\section{Competing interests}

The authors have no competing interest to declare.
Received: 30 September 2019 Accepted: 19 November 2019

Published online: 04 December 2019

References

1. Pihlstrom BL, Michalowicz BS, Johnson NW. Periodontal diseases. Lancet. 2005;366(9499):1809-20.

2. Lovegrove JM. Dental plaque revisited: bacteria associated with periodontal disease. J N Z Soc Periodontol. 2004;87:7-21.

3. Darveau RP, Hajishengallis G, Curtis MA. Porphyromonas gingivalis as a potential community activist for disease. J Dent Res. 2012;91(9):816-20.

4. Hajishengallis G. Immunomicrobial pathogenesis of periodontitis: keystones, pathobionts, and host response. Trends Immunol. 2014;35(1):3-11.

5. Hajishengallis G, Darveau RP, Curtis MA. The keystone-pathogen hypothesis. Nat Rev Microbiol. 2012;10(10):717-25.

6. Hajishengallis G, Lamont RJ. Beyond the red complex and into more complexity: the polymicrobial synergy and dysbiosis (PSD) model of periodontal disease etiology. Mol Oral Microbiol. 2012;27(6):409-19.

7. Hajishengallis G, Lamont RJ. Breaking bad: manipulation of the host response by Porphyromonas gingivalis. Eur J Immunol. 2014;44(2):328-38.

8. Hajishengallis G, Lamont RJ. Dancing with the stars: how choreographed bacterial interactions dictate nososymbiocity and give rise to keystone pathogens, accessory pathogens, and pathobionts. Trends Microbiol. 2016; 24(6):477-89.

9. Lamont RJ, Hajishengallis G. Polymicrobial synergy and dysbiosis in inflammatory disease. Trends Mol Med. 2015;21(3):172-83.

10. Maekawa T, Krauss JL, Abe T, Jotwani R, Triantafilou M, Triantafilou K, et al. Porphyromonas gingivalis manipulates complement and TLR signaling to uncouple bacterial clearance from inflammation and promote dysbiosis. Cell Host Microbe. 2014;15(6):768-78.

11. Genco RJ, Van Dyke TE. Prevention: reducing the risk of CVD in patients with periodontitis. Nat Rev Cardiol. 2010;7(9):479-80.

12. Han YW, Wang X. Mobile microbiome: oral bacteria in extra-oral infections and inflammation. J Dent Res. 2013;92(6):485-91.

13. Lundberg K, Wegner N, Yucel-Lindberg T, Venables PJ. Periodontitis in RAthe citrullinated enolase connection. Nat Rev Rheumatol. 2010;6(12):727-30.

14. Madianos PN, Bobetsis YA, Offenbacher S. Adverse pregnancy outcomes (APOs) and periodontal disease: pathogenic mechanisms. J Clin Periodontol. 2013;40(Suppl 14):S170-80.

15. Whitmore SE, Lamont RJ. Oral bacteria and cancer. PLoS Pathog. 2014;10(3): e1003933.

16. Habashneh RA, Khader YS, Alhumouz MK, Jadallah K, Ajlouni Y. The association between inflammatory bowel disease and periodontitis among Jordanians: a case-control study. J Periodontal Res. 2012;47(3):293-8.

17. Brito F, de Barros FC, Zaltman C, Carvalho AT, Carneiro AJ, Fischer RG, et al. Prevalence of periodontitis and DMFT index in patients with Crohn's disease and ulcerative colitis. J Clin Periodontol. 2008;35(6):555-60. 
18. Xavier RJ, Podolsky DK. Unravelling the pathogenesis of inflammatory bowel disease. Nature. 2007;448(7152):427-34.

19. Rautava J, Pinnell LJ, Vong L, Akseer N, Assa A, Sherman PM. Oral microbiome composition changes in mouse models of colitis. J Gastroenterol Hepatol. 2015;30(3):521-7.

20. Arimatsu K, Yamada H, Miyazawa H, Minagawa T, Nakajima M, Ryder Ml, et al. Oral pathobiont induces systemic inflammation and metabolic changes associated with alteration of gut microbiota. Sci Rep. 2014;4:4828.

21. Nakajima M, Arimatsu K, Kato T, Matsuda Y, Minagawa T, Takahashi N, et al. Oral Administration of $P$. gingivalis induces dysbiosis of gut microbiota and impaired barrier function leading to dissemination of enterobacteria to the liver. PLoS One. 2015;10(7):e0134234.

22. Peterson LW, Artis D. Intestinal epithelial cells: regulators of barrier function and immune homeostasis. Nat Rev Immunol. 2014;14(3):141-53.

23. Clevers H, Loh KM, Nusse R. Stem cell signaling. An integral program for tissue renewal and regeneration: Wnt signaling and stem cell control. Science. 2014;346(6205):1248012.

24. Kim HS, Shin TH, Lee BC, Yu KR, Seo Y, Lee S, et al. Human umbilical cord blood mesenchymal stem cells reduce colitis in mice by activating NOD2 signaling to COX2. Gastroenterology. 2013;145(6):1392-1403.e1-8.

25. Kim HS, Shin TH, Yang SR, Seo MS, Kim DJ, Kang SK, et al. Implication of NOD1 and NOD2 for the differentiation of multipotent mesenchymal stem cells derived from human umbilical cord blood. PLoS One. 2010;5(10): e15369.

26. Nigro G, Rossi R, Commere PH, Jay P, Sansonetti PJ. The cytosolic bacterial peptidoglycan sensor Nod2 affords stem cell protection and links microbes to gut epithelial regeneration. Cell Host Microbe. 2014;15(6):792-8.

27. Zhao JL, Ma C, O'Connell RM, Mehta A, DiLoreto R, Heath JR, et al. Conversion of danger signals into cytokine signals by hematopoietic stem and progenitor cells for regulation of stress-induced hematopoiesis. Cell Stem Cell. 2014;14(4):445-59

28. Sato T, Vries RG, Snippert HJ, van de Wetering M, Barker N, Stange DE, et al. Single Lgr5 stem cells build crypt-villus structures in vitro without a mesenchymal niche. Nature. 2009;459(7244):262-5.

29. Blasco-Baque V, Garidou L, Pomie C, Escoula Q, Loubieres P, Le Gall-David S, et al. Periodontitis induced by Porphyromonas gingivalis drives periodontal microbiota dysbiosis and insulin resistance via an impaired adaptive immune response. Gut. 2017:66(5):872-85

30. Saito K, Katakura K, Suzuki R, Suzuki T, Ohira H. Modulating toll-like receptor 4 signaling pathway protects mice from experimental colitis. Fukushima J Med Sci. 2013;59(2):81-8

31. Lee J, Mo JH, Katakura K, Alkalay I, Rucker AN, Liu YT, et al. Maintenance of colonic homeostasis by distinctive apical TLR9 signalling in intestinal epithelial cells. Nat Cell Biol. 2006;8(12):1327-36.

32. Rachmilewitz D, Karmeli F, Takabayashi K, Hayashi T, Leider-Trejo L, Lee J, et al. Immunostimulatory DNA ameliorates experimental and spontaneous murine colitis. Gastroenterology. 2002;122(5):1428-41.

33. Rakoff-Nahoum S, Paglino J, Eslami-Varzaneh F, Edberg S, Medzhitov R. Recognition of commensal microflora by toll-like receptors is required for intestinal homeostasis. Cell. 2004;118(2):229-41.

34. Hirschfeld M, Weis JJ, Toshchakov V, Salkowski CA, Cody MJ, Ward DC, et al. Signaling by toll-like receptor 2 and 4 agonists results in differential gene expression in murine macrophages. Infect Immun. 2001;69(3):1477-82.

35. Kirikae T, Nitta T, Kirikae F, Suda Y, Kusumoto S, Qureshi N, et al. Lipopolysaccharides (LPS) of oral black-pigmented bacteria induce tumor necrosis factor production by LPS-refractory $\mathrm{C} 3 \mathrm{H} / \mathrm{HeJ}$ macrophages in a way different from that of Salmonella LPS. Infect Immun. 1999;67(4):1736-42.

36. Ogawa T, Asai Y, Makimura Y, Tamai R. Chemical structure and immunobiological activity of Porphyromonas gingivalis lipid A. Front Biosci. 2007;12:3795-812.

37. Hirschfeld M, Ma Y, Weis JH, Vogel SN, Weis JJ. Cutting edge: repurification of lipopolysaccharide eliminates signaling through both human and murine toll-like receptor 2. J Immunol. 2000;165(2):618-22.

38. Nativel B, Couret D, Giraud P, Meilhac O, d'Hellencourt CL, Viranaicken W, et al. Porphyromonas gingivalis lipopolysaccharides act exclusively through TLR4 with a resilience between mouse and human. Sci Rep. 2017;7(1):15789.

39. Andrukhov O, Ertlschweiger S, Moritz A, Bantleon HP, Rausch-Fan X Different effects of $P$. gingivalis LPS and E. coli LPS on the expression of interleukin-6 in human gingival fibroblasts. Acta Odontol Scand. 2014;72(5): 337-45.
40. Naito T, Mulet C, De Castro C, Molinaro A, Saffarian A, Nigro G, et al. Lipopolysaccharide from crypt-specific core microbiota modulates the colonic epithelial proliferation-to-differentiation balance. MBio. 2017:8(5): 01680-17.

41. Kim H, Jeong $H$, Cho $Y$, Lee J, Nam KT, Lee HW. Disruption of the Tff1 gene in mice using CRISPR/Cas9 promotes body weight reduction and gastric tumorigenesis. Lab Anim Res. 2018;34(4):257-63.

42. Kim JI, Park JS, Kim H, Ryu SK, Kwak J, Kwon E, et al. CRISPR/Cas9-mediated knockout of Rag-2 causes systemic lymphopenia with hypoplastic lymphoid organs in FVB mice. Lab Anim Res. 2018;34(4):166-75.

43. Lee H, Kim JI, Park JS, Roh Jl, Lee J, Kang BC, et al. CRISPR/Cas9-mediated generation of a Plac8 knockout mouse model. Lab Anim Res. 2018;34(4): 279-87.

\section{Publisher's Note}

Springer Nature remains neutral with regard to jurisdictional claims in published maps and institutional affiliations.

\section{Ready to submit your research? Choose BMC and benefit from:}

- fast, convenient online submission

- thorough peer review by experienced researchers in your field

- rapid publication on acceptance

- support for research data, including large and complex data types

- gold Open Access which fosters wider collaboration and increased citations

- maximum visibility for your research: over $100 \mathrm{M}$ website views per year

At BMC, research is always in progress.

Learn more biomedcentral.com/submissions 